\title{
Automation of Isolated Diagnosis Faults by Coupling Vibration Analysis- Artificial neural networks.
}

\author{
N. Menasri ${ }^{1 *}$, A. Bouchoucha ${ }^{2}$, D. Khoja ${ }^{3}$, M. Zaoui ${ }^{4}$ \\ 1,3,4 Mechanics Laboratory, Faculty of Technology, University of M'sila PO Box 166 M'sila 28000 Algeria \\ ${ }^{2}$ Tribology Laboratory, Faculty of Technology, University of Maintouri Constantine 25000 Algeria
}

\section{KEYWORDS}

Wear, bearings, gear, vibration analysis, artificial neural networks and diagnosis.

\begin{abstract}
Rotating machines play a strategic role in a manufacturing process; it is the case of a cement mill. These machines are made of fragile organs (bearings and gears, etc.) subjected to significant mechanical stresses and harsh industrial environments. Improving productivity through better control of the production tool through its automation, although by controlling its availability; automation must be associated with a maintenance strategy that will ensure a more availability. However, many techniques available currently require much expertise to successfully implement; it requires new techniques that allow relatively unskilled operators to make reliable decisions without knowing the mechanism of system and analyzing the data. The artificial neural networks (ANN) are suitable for this type of problem diagnosis using the classification method.

This article discusses the automation of isolated diagnosis faults of bearings and gears in a gear unit DMGH25.4 of cement mill by coupling spectral analysis vibration-neural networks.
\end{abstract}

\section{Introduction}

Bearings and gears are strategic components of rotating machinery, they ensure the transmission of mechanical forces and the rotation of the shaft, but they are the most fragile. This type of transmission main vibrates which shocks produced by contacting of gear teeth.

In practice, a wholesome gearing is not ideal due to geometric differences, and has a spectrum consisting of gearing harmonics. When the two wheels has a tooth deteriorated, there is a shock to the periodic frequency of rotation of the wheel concerned, the corresponding spectrum shows not only the frequency of gearing above, but also a comb rays whose pitch corresponding at this frequency of rotation [1]-[3], [7]-[9], [11], [12].

A new bearing in excellent working condition produces low amplitude vibrations looks to random noise. During the apparition of damage, a pulse occurs each time the defect participates in contact. The damage has a frequency characteristic which depends on the geometry of the bearing and the location of spalling (on the inner ring, outer ring, or the ball) [12]. However, the amplitudes of the vibrations induced defects are not very high. They are embedded among the most energy system components such as gears or those related to an imbalance. The spectrum of an acceleration mea- 
surement of a bearing with a defective bearing has a higher level in the high frequency band (of $1 \mathrm{KHz}$ to $10 \mathrm{KHz}$ ), and the sidebands around frequencies predominate (modulation phenomena on signals) [1], [11].

Therefore these vibrations occupy a privileged place among the parameters to be considered in making a diagnosis. Whenever a fault participates in a contact, change the vibration characteristics of the system. By analyzing the acceleration measurements from an accelerometer, we can identify and quantify these changes in order to establish a relationship between the measurements and the type of fault, which will use as signatures for fault diagnosis system.

However, many techniques available presently require monitoring of much expertise to apply them successfully. It requires new techniques that allow relatively unskilled operators to make reliable decisions without knowing the mechanism of system and analyze the data. The literature review showed the effectiveness of artificial neural networks using the classification method to meet this function [9]. Classifications can be produced either entirely from characteristics of experimental signals, either from models generated by the network to provide an answer. In the category of networks operates from experimental signals exist multilayer networks. For them, the forms extracted from mea-

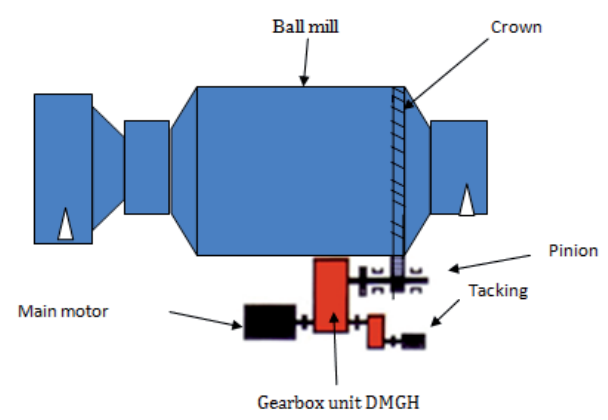

Fig. 1: Description of cement mill DMGH25.4

It consists of a main motor; a tacking; a grinder DMGH; a gear unit horizontal.

\subsection{Gear unit DMGH 25.4}

Fig. 2 shows the gear unit Flender DMGH25.4, which driven by a main motor through elastic coupling RUPLEX RLS 800. In order to move the grinder sures must be carefully chosen to be representative of the diagnostic information [4], [5], [10], [13], [14].

In this paper we deal with the application of artificial neural networks for automated fault diagnosis isolated bearings and gears in a gear unit DMGH25.4 of a cement mill. In section II we describe the system. In section III we present the measures companions. In section IV we present vibration analysis illustrating the influence of defects on the structure of signals. Finally, in section $V$ we present the application of artificial neural networks ANN for isolated diagnosis faults of bearings and gears system.

\section{Description of the System 2.1 Description of cement mill}

The cement mill flender DMGH of the Algerian cement company ACC is a horizontal ball mill, driven by lateral crown fig. 1. It receives the clinker, gypsum and possibly additional materials; loading is performed by a bucket chain to the input of the machine. This mixture is generally pre-crushed with captured material and repressed by the separator, using steel balls placed inside the rotating grinder; then the whole is supported by an endless screw, an elevator or another transport device, to be processed downstream [6].

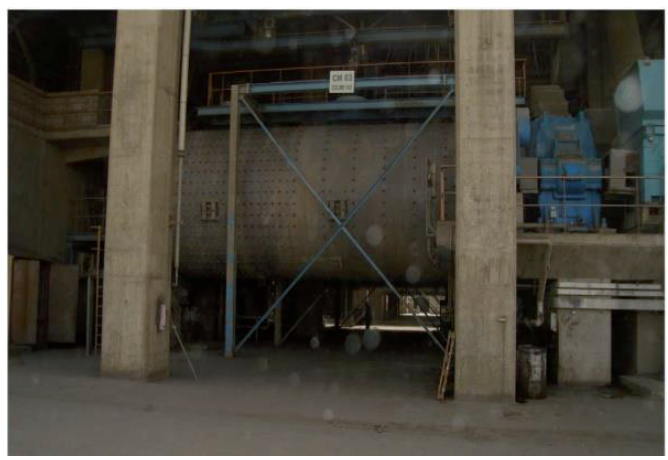

on a desired position, the gear unit is connected to a tacking with a motor (auxiliary motor) and a brake capable of blocking the grinder on any position. The kinematic characteristics of the gear unit are [6]:

- The speed of the high speed shaft is $16.57 \mathrm{~Hz}$.

- The speed of the intermediate shaft is $7.27 \mathrm{~Hz}$. 
- The speed of the output shaft is $2.05 \mathrm{~Hz}$.

- G9H speed grinder is $0.256 \mathrm{~Hz}$.

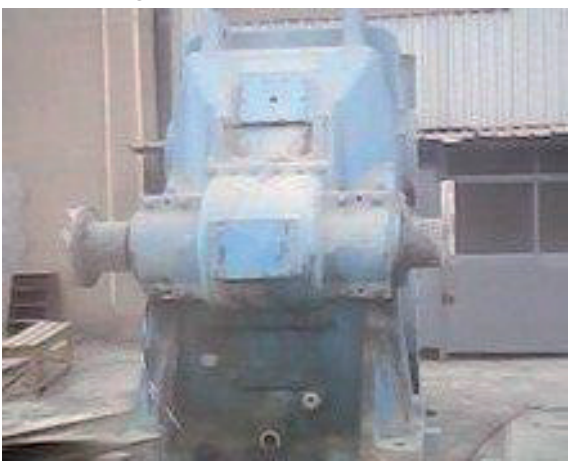

Table1 presents the technical data of the gear unit [6].

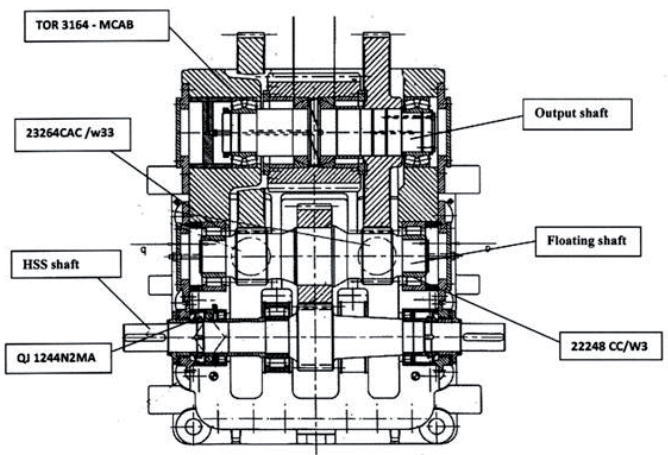

Fig. 2: Gear unit flender DMGH 25.4

Table 1: Technical data of the gear unit

\begin{tabular}{|c|c|c|c|}
\hline Mark & Type & Module (mm) & Power (KW) \\
\hline Flender & DMGH 25.4 & 25.4 & 4551 \\
\hline Speed (T/mn) & Weight (Kg) & $\begin{array}{l}\text { Report teeth input shaft / } \\
\text { intermediate }\end{array}$ & $\begin{array}{l}\text { Report teeth intermediate } \\
\text { shaft / output }\end{array}$ \\
\hline $990 / 122.30$ & 35000 & $25 / 57$ & $20 / 71$ \\
\hline $\begin{array}{l}\text { Report teeth } \\
\text { pinion / crown }\end{array}$ & $\begin{array}{l}\text { Bearings of High speed } \\
\text { shaft HSS }\end{array}$ & Bearings of output shaft & $\begin{array}{l}\text { Bearings of intermediate } \\
\text { shaft }\end{array}$ \\
\hline $31 / 248$ & $\begin{array}{l}\text { 2×22248 CC / N1W33C3 } \\
\text { QJ1244N2MA }\end{array}$ & $\begin{array}{l}2 \times 23264 \text { CAC / W33 } \\
\text { 2×TOR F3164-MCAB }\end{array}$ & 2XTOR F3164-G \\
\hline \multicolumn{2}{|c|}{$\begin{array}{l}\text { 3. Companions Measures } \\
\text { This study interest in the isolated faults bearings } \\
\text { and gears (in our study we limit ourselves to fo- } \\
\text { cus on the wear of the outer ring of the bearing } \\
\text { QJ1244 N2MA and a tooth deterioration of the in- } \\
\text { termediate pinion). } \\
\text { To analyze the vibrations generated by the organs } \\
\text { of the gear unit, the measurements were carried } \\
\text { out in three directions (axial, horizontal, vertical) in } \\
\text { eight points' shaft bearing using an accélomètre } \\
\text { A0760GP SNP66223. Signal acquisition was made } \\
\text { by a measuring device CSI } 2130 \text { machinery health } \\
\text { vibration analysis (Fig. 3), with a sampling time }\end{array}$} & 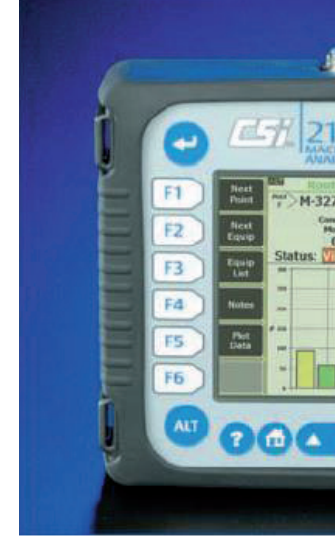 & 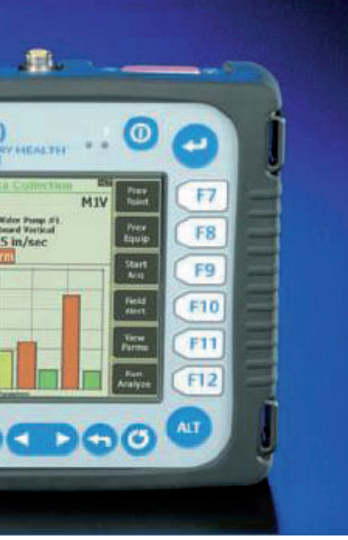 \\
\hline
\end{tabular}

$\mathrm{Te}=0.78 \mathrm{~Hz}$. This device not allows only receiving signal from the accelerometer to process the signal or the spectrum calculating acceleration, but also to perform the integration to obtain the information in the speed mode. Spectral analysis was done using the AMS Suite.

Table 2 gives the characteristics of SKF bearing QJ 1244 N2MA.

Fig. 3: CSI 2130 analyzer.

Table 2: Characteristics of SKF bearing QJ 1244 N2MA

\begin{tabular}{|l|l|l|l|}
\hline $\begin{array}{l}\text { Number of } \\
\text { balls }\end{array}$ & $\begin{array}{l}\text { Ball } \\
\text { diameter }\end{array}$ & $\begin{array}{l}\text { Piste } \\
\text { diameter }\end{array}$ & $\begin{array}{l}\text { Contact } \\
\text { angle }\end{array}$ \\
\hline 15 & $57.15 \mathrm{~mm}$ & $310 \mathrm{~mm}$ & $45^{\circ}$ \\
\hline
\end{tabular}


After calculation, the frequencies typical defects SKF QJ 1244 N2MA (Table 3) and meshing is [1]:

Table 3: The frequencies typical defects SKF QJ 1244 N2MA

\begin{tabular}{|c|c|c|}
\hline \multicolumn{2}{|c|}{ Component fault } & Fault frequency $(\mathrm{Hz})$ \\
\hline FC & shaft 1-IB & 7,20 \\
\hline FRB & shaft 1-IB & 44,17 \\
\hline FEPB & shaft 1-IB & 100,85 \\
\hline FIPB & shaft 1-IB & 131,08 \\
\hline
\end{tabular}

Where:

FC: fault cage;

FSB: fault ball

FEPB: fault outer ring;

FIPB: fault inner ring.

Meshing frequency shaft 1/ Shaft 2: 414, $17 \mathrm{~Hz}$ Meshing frequency shaft 2 / Shaft 3: 145, $32 \mathrm{~Hz}$ Meshing frequency shaft 2 / Shaft 4: 145, $32 \mathrm{~Hz}$ Meshing frequency shaft 3 and crown $63.45 \mathrm{~Hz}$.

\section{Vibration Analyses}

Measurements of Level 1 (RMS) are not always sufficient for faults location. To locate faults, the spectra points will be assessed in three directions. Fig. 4 shows the vibration spectrum of the image of point 2 along the three directions in the frequency range $[0-10000 \mathrm{KH}]$ with acceleration $(\mathrm{g})$.
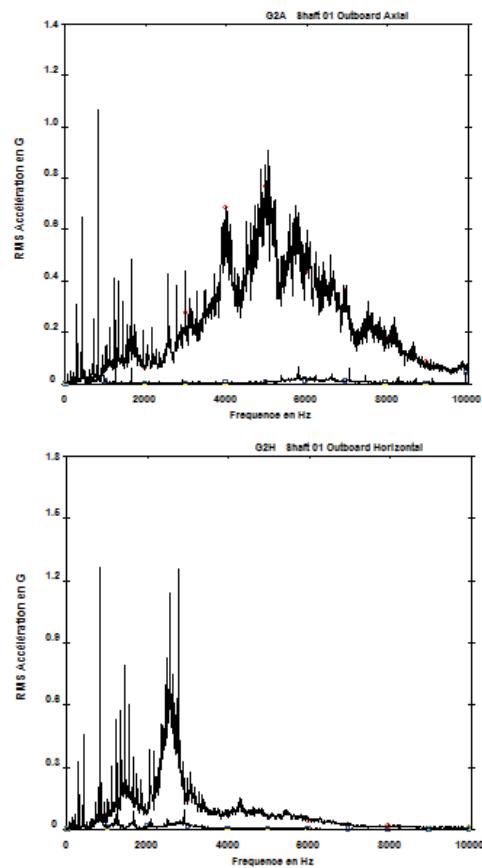

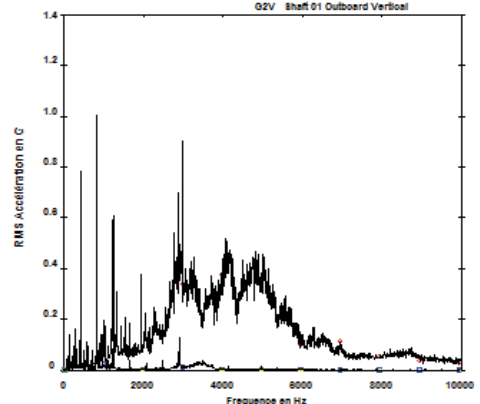

Fig. 4: Spectrum signals of point 2 with and without fault

Fig. 5 shows the spectrum envelope with and without fault, which allow determining the kinematic characteristic of the fault.
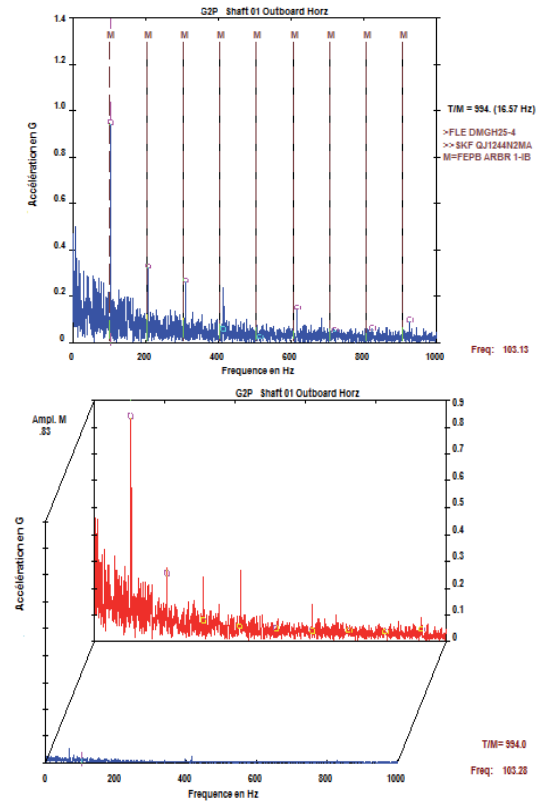

Fig. 5: Spectrum envelope vibration signals of point 2 with and without fault.

We observe that the specters are flattened in the absence of fault; in contrast, we observe that peaks shocks are the presence of the defect. The fault is due to the wear of the bearing outer ring QJ1244 N2MA, because we observe peaks shocks at high frequencies close to the frequency of passage of the outer race defect $M=$ FEPB shaft 1-IB.

It is noted that reasoning on the signal amplitude, 
the signature of bearing faults do not propagate across the entire mechanical system. The faults of bearing cause an increase of the acceleration amplitude in the bearing where they are housed. Indeed, in the case of important defects, their effect may affect neighboring bearings driven by the same shaft at very high speeds. The accelerations measured on another shaft (in association with the support shaft gear fault rolling) are not affected by the damage.

The following figure shows a fault meshing (deterioration of a tooth of the pinion of the intermediate shaft (horizontal measurement point 4)).

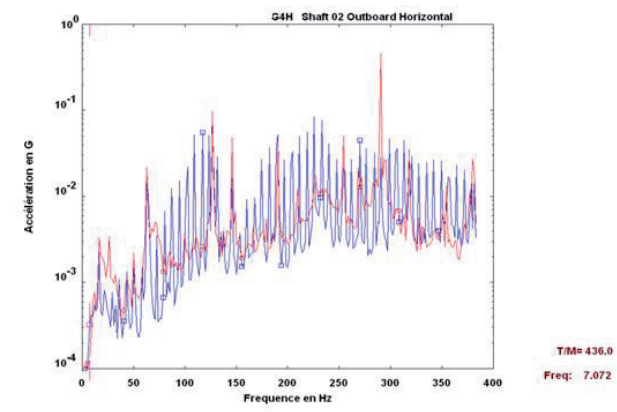

Fig. 6: Spectrum signal of point 4 with and without fault.

The signal shows peaks representing the participation of the damaged tooth meshing.

\section{Automation Diagnosis Using Neural Networks}

In the following, we apply the approach of neural networks on a set of real measurement data QJ1244 ball bearing and pinion of the intermediate shaft with or without faults (wear of the outer ring and fracture of a tooth of the intermediate pinion).

\subsection{Construction of the block of ANN}

The neural network we have chosen is a network that uses multilayer retro propagation algorithm for learning. This method gave good results in many applications [4]. To apply it suffices to have the input data and output data.

Stages of construction and validation of the neural network are divided into three phases:

\subsubsection{Choice of network inputs}

The selected inputs are the eight amplitude values of the spectrum of accelerations in three directions for point 2 and the eight values in the hori- zontal direction for the point 4; the latter has 32 inputs of the input layer that are sampled values of acceleration specters (Fig. 7).

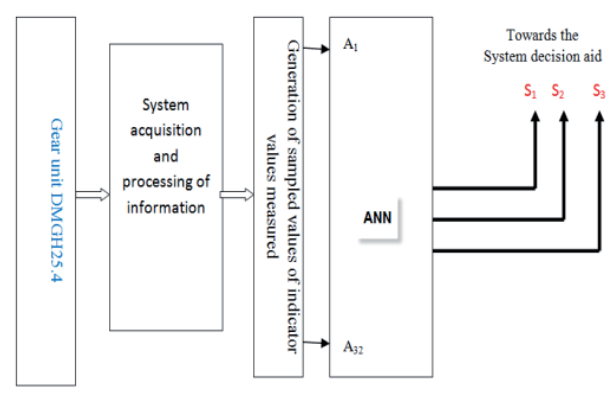

Fig. 7: Diagram of the diagnostic system by neural network.

\subsubsection{Choice of network outputs}

Our network has three outputs because in our case we have been considering faults are not many (see Fig. 7). We associate each fault a code, i.e. each fault is represented by the three output neurons (see Table 4).

When detecting of a fault, the network must indicate any binary number (e.g. 100) at its output, which corresponds to this type of fault (wear of the outer ring bearing QJ1244 N2MA). In other word each output of the network has a single digit is 1 or 0 .

Table 4: Classification of types of faults horizontal mill DMGH 25.4.

\begin{tabular}{|l|l|l|l|l|l|l|l|l}
\hline Category & Type of fault Symbol & Code \\
& & S1 S2 S3
\end{tabular}

\begin{tabular}{|l|l|l|lll|}
\hline 1 & Faultless & NF & 0 & 0 & 0 \\
\hline 2 & $\begin{array}{l}\text { wear of the } \\
\text { outer ring } \\
\text { QJ1244 }\end{array}$ & WBQJ1244 & 1 & 0 & 0 \\
\hline 3 & $\begin{array}{l}\text { deteriora- } \\
\text { tion of a } \\
\text { tooth of the } \\
\text { intermedia- } \\
\text { te pinion }\end{array}$ & DTIP & 0 & 1 & 0 \\
\hline
\end{tabular}

5.1.3 Learning and test neural network selected

The network used is a multi-layer network (Fig. 8), comprising an input layer corresponding to the retina, an output layer corresponding to the decision, and a hidden layer. The number of neurons in each layer is given by Table 5. The selected network 
is entrained by the retro propagation algorithm.

Table 5: Number of neurons in each layer.

\begin{tabular}{|l|c|c|c|}
\hline $\begin{array}{l}\text { Network Input layer } \\
\text { constructed }\end{array}$ & Hidden layer & Output layer \\
\hline $\begin{array}{l}\text { Numbers of } \\
\text { neurons }\end{array}$ & 20 & 4 & 3 \\
\hline
\end{tabular}

We made an automatic learning using MATLAB (SIMULINK) until the smaller squared error. The mean square error is the smallest obtained after 19 iterations equal 2.0633E-11 (Fig. 9).

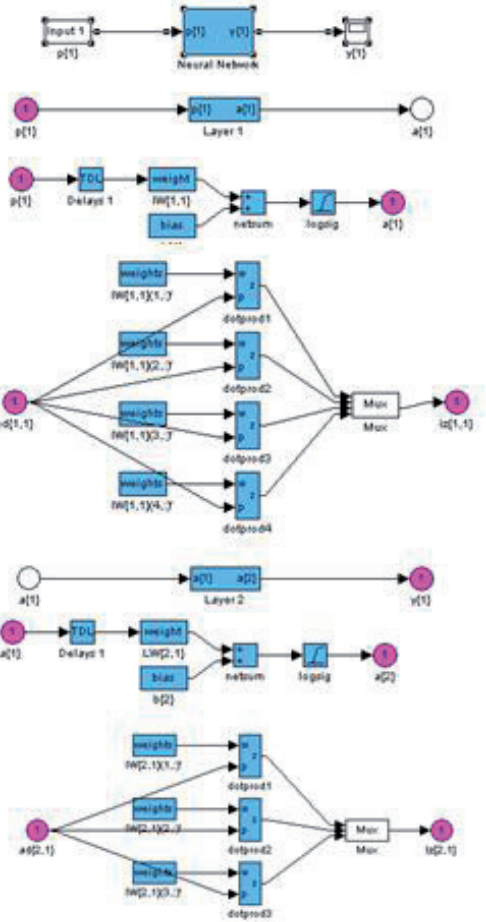

Fig. 8: Structure of the network selected.

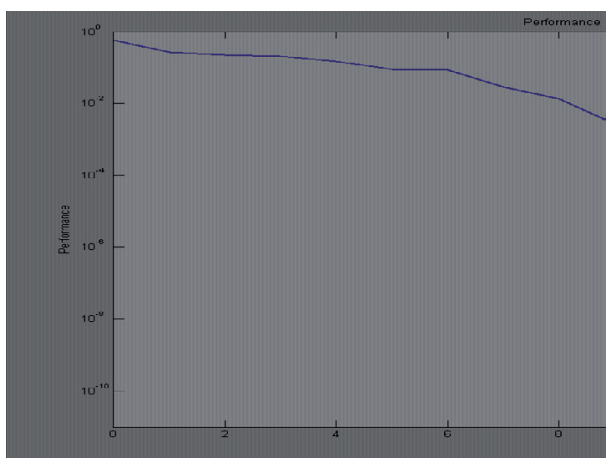

Fig. 9: Diagram of the diagnostic system by neural network.
Once the neural network is constructed and its learning achieved satisfactory performance, we moves to step test by examples to the input of the network In fact, these examples belong to two databases, the first being the learning base and the second is based on tests which we proceed to test the network capacity to recognize examples not learned. This last operation is used to estimate the capacity generalization of the network (see Fig. 10). The tests are performed according to the following procedure: sane system, then default 1, sane system, then default 2 , sane system, then fault test not learned, and that for a period of time $2 \mathrm{~s}$ for each test.

It is evident that the tests of the neural network on the learned examples (Fig. 11) have given better results, because all types of running (anomalies and normal running) were identified exactly by the network, this can be explained by the results obtained
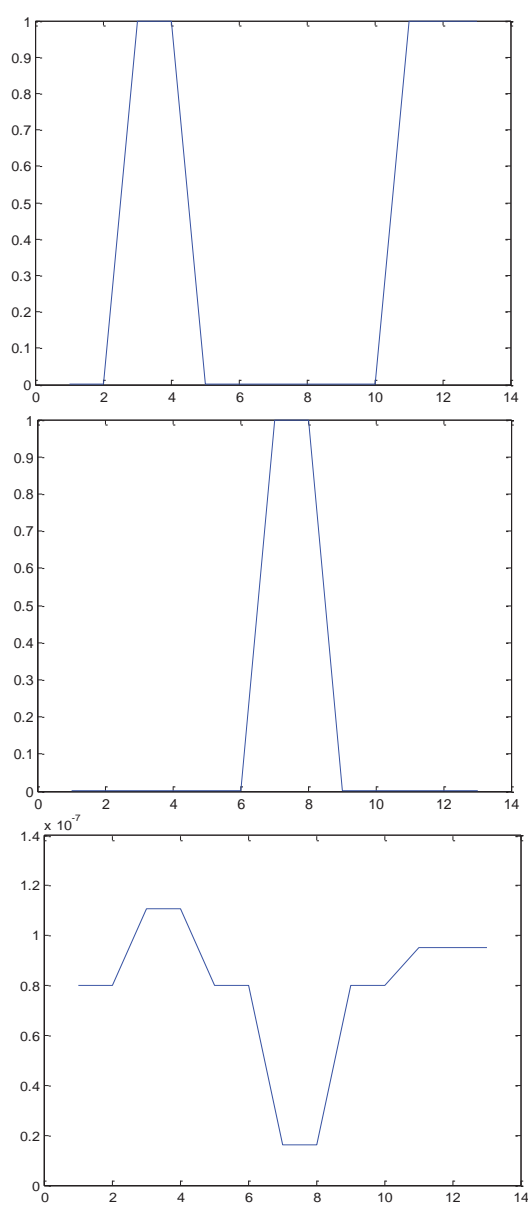

Fig. 11: Graphical representation testing the Artificial Neural Network (ANN). 
in the learning phase of the network (including the value of the mean square error is close to zero).

Regarding the network test on examples that have not been learned in the learning phase, we tested the network by a signal due to wear of the outer ring QJ 1244, its result is presented in fig. 10.

From the results obtained in the testing phase, we found that the outputs of the network evolve according to the desired output predetermined before.

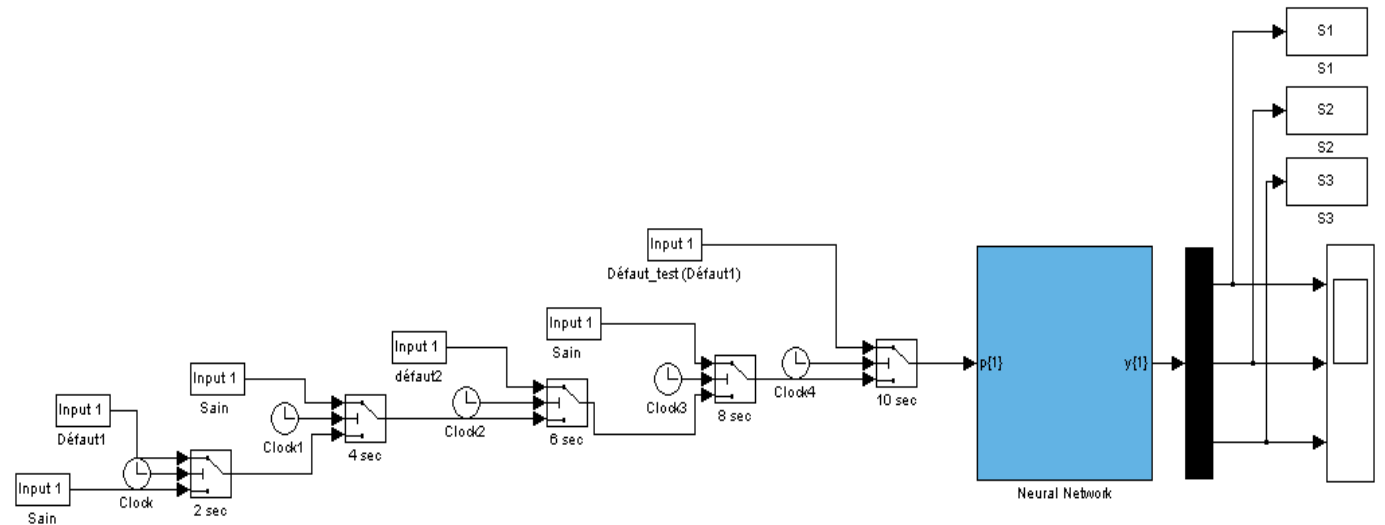

Fig. 10: Simulation test Neural Network.

\section{Conclusion}

Measures of Level 1 (RMS) are not always sufficient for fault location. To locate faults, the spectral analyses of the measurement points were made:

- In presence of a fault of the bearing outer ring we observe peaks shock at high frequencies near the frequency of the passage of the defect of the outer ring.

- In presence of a fault gear (deterioration of a tooth) shows the corresponding spectrum of a comb lines which the pitch corresponds to the rotational frequency.

The difficulty of interpretation of a form, value, makes delicate operations of monitoring. The automation of this process by the neural network hidden layer with retro propagation learning gave correct results. This work has validated the performance of neural networks for a classification problem.

\section{Acknowledgements}

This work was supported by Algeria Cement Company ACC and MEI.

\section{References}

[1] Ahmadi H., Mollazada.K., Bearing fault diagnosis of a mine stone crasher by vibration condition monitoring technique, Res.J.Appl.Sci.Eng.Technol, vol 1(3), 2009, p. 112-115.

[2] Dyer D., Stewart R.M., Detection of rolling element bearing damage by statistical analysis, ASME journal of mechanical design, $n^{\circ} 100,1978$, p. 229-235.

[3] Garreau D., Monitoring of the bearing by vibration analysis, cetim information, $n^{\circ} 115,1990$.

[4] Khodja DJ., Chetate B., Development of Neural Network module for fault identification in Asynchronous machine using various types of reference signals, 2nd International Conference Physics and Control, August 24-26 2006, St Petersburg, Russia, p. 537-542.

[5] Kolodziejczyk T., al., Artificial intelligence as efficient technique for ball bearing fretting wear damage prediction, Wear, vol 268, 2010, p. 309-315.

[6] Manual cement mill Flender.

[7] McFadden P.D., Detection fatigue cracks in gears by amplitude and phase demodulation of Meshing vibration, ASME Transaction Journal of Vibration Acoustics and Reliability in Design, vol 108, 1986 p. 165-170.

[8] Mol H.A., Rolling bearing localized defect detection through vibration envelope analysis, SKF Engineering and Research centre BV, 2000, Sweden.

[9] Monk R., Vibration measurement gives early warning of mechanical faults, processing engineering, 1997.

[10] Patrick H., Simpson K., Foundations of neural network, Technology Update series, IEEE, 1996, p. 1-20.

[11] Randall R.B, Antoni J., Rolling element bearing diagnosticsA tutorial, Mechanical systems and signal processing 25, 2011, p. 485-520.

[12] Trajin B., Automatic detection and diagnosis of bearing defects in an asynchronous machine by spectral analysis of stator currents, JCGE'08 Lyon, 16-17 December 2008.

[13] Wang $H_{\text {., }}$ Chen P., Intelligent diagnosis method for rolling element bearing faults using possibility theory and neural 
Acta Mechanica Slovaca

Journal published by Faculty of Mechanical Engineering - Technical University of Košice

network, Computers \& industrial engineering, vol 60, 2011, p. 511-518,

[14] Yang D.M., Stronach A.F., P. MacConnell., Third order spectral techniques for the diagnosis of motor bearing condition using artificial neural networks, Mechanical Systems and Signal Processing 16(2-3), 2002, p. 391-411. 